\title{
Routing Efficiency of AODV and DSR Protocols in Ad-Hoc Sensor Networks
}

\author{
Tao Yang†, Makoto Ikeda†, Giuseppe De Marcoł, Leonard Barolli ††, \\ Arjan Durresił†, Fatos Xhafa \\ †Graduate School of Engineering \\ Fukuoka Institute of Technology (FIT) \\ 3-30-1 Wajiro-Higashi, Higashi-Ku, Fukuoka 811-0295, Japan \\ E-mail: $\{$ bd07003,bd07001\}@ws.ipc.fit.ac.jp \\ $\ddagger$ Department of Systems and Information Engineering \\ Toyota Technological Institute \\ 2-12-1 Tenpaku-Hisakata, Nagoya 468-8511, Japan \\ E-mail:demarco@toyota-ti.ac.jp \\ ††Department of Information and Communication Engineering \\ Fukuoka Institute of Technology (FIT) \\ 3-30-1 Wajiro-Higashi, Higashi-Ku, Fukuoka 811-0295, Japan \\ E-mail: barolli@fit.ac.jp \\ ††Department of Computer and Information Science \\ Indiana University Purdue University Indianapolis \\ 723 W. Michigan Street SL 280 \\ Indianapolis, IN 46202, USA \\ E-mail: durresi@cs.iupui.edu \\ 廿Department of Languages and Informatics Systems \\ Polytechnic University of Catalonia \\ C/Jordi Girona 1-3, 08034 Barcelona, Spain \\ E-mail: fatos@1si.upc.edu
}

\begin{abstract}
In this paper, we consider the performance behavior of two protocols: AODV and DSR using TwoRayGround model. We study the routing efficiency of the ad-hoc sensor network and compare the performance of two protocols for different scenarios using the routing efficiency concept. By computer simulations, we found that the routing efficiency of DSR protocol is better than AODV protocol.
\end{abstract}

\section{Introduction}

In recent years, technological advances have lead to the emergence of distributed Wireless Sensor and Actor Networks (WSANs) which are capable of observing the physical world, processing the data, making decisions based on the observations and performing appropriate actions. These networks can be an integral part of systems such as battlefield surveillance and microclimate control in buildings, nuclear, biological and chemical attack detection, home automation and environmental monitoring.

In WSANs, the phenomena of sensing and acting are performed by sensor and actor nodes, respectively. However, in some applications instead of actor nodes, integrated sensor/actor nodes which include both sensing and acting units can also be used. One of the examples of this kind of application is the distributed robot system. In such system, robots which have both sensing and acting capabilities function as integrated sensor actor nodes $[1,2]$.

Different from Wireless Sensor Networks (WSNs) where the communication takes place between sensors and the sink, in WSANs, new networking phenomenons called sensor-actor and actor-actor communications may occur. 
Sensor-actor communication provides the transmission of event features from sensors to actors. After receiving event information, actors need to communicate with each other in order to perform the appropriate action on the event area.

Recently, we witnessed a lot of research effort towards the optimization of standard communication paradigms for WSN. In fact, the traditional Wireless Network (WN) design has never paid attention to constraints such as the limited or scarce energy of nodes and their computational power. Another aspect which is different from traditional WN is the communication reliability and congestion control. In traditional wired nets, one reasonably supposes that communication paths are stable along the transmission instances. This fact permits to use the end-to-end approach to the design of reliable transport and application protocols. The TCP works well because of the stability of links. On the other hand, in WSN and WSAN paths can change over time, because of time-varying characteristics of links and nodes reliability. These problems are important especially in a multi-hop scenario, where nodes accomplish also at the routing of other nodes' packets. Ad-hoc routing and new MAC protocols should be used to accommodate the requirements of the ad-hoc WSN.

In this paper, we study the behavior of two routing protocols: AODV and DSR using TwoRayGround radio model and compare the performance of two protocols based on the concept of Routing Efficiency (RE). As a technique for congestion control in our model, we use the packet repetition transmission. The repetition rate depends on a number of other factors, first of all the bound on the signal distortion perceived at the sink node.

The paper is organized as follows. In Section 2, we explain the proposed network simulation model. In Section 3, we present routing protocols. In Section 4, we discuss the RE concept. In Section 5, we show the simulation results. Finally, the conclusions are given in Section 6.

\section{Proposed Network Simulation Model}

Proposed network simulation model is shown in Fig. 1 and the sensor node model is shown in Fig. 2. In Fig. 2, the channel 1 is used for neighbor nodes communication and channel 2 for communication between present node and event node.

In our ad-hoc WSN, every node detects the physical phenomenon and sends back to the actor node data packets. We suppose that the actor node is more powerful than sensor nodes. This model can be used for remote monitoring of hazard or inaccessible areas [3]. We analyze the performance of the network in a fixed time interval, $\tau$. This can be considered as the available time for the detection of the phenomenon and its value is application dependent.

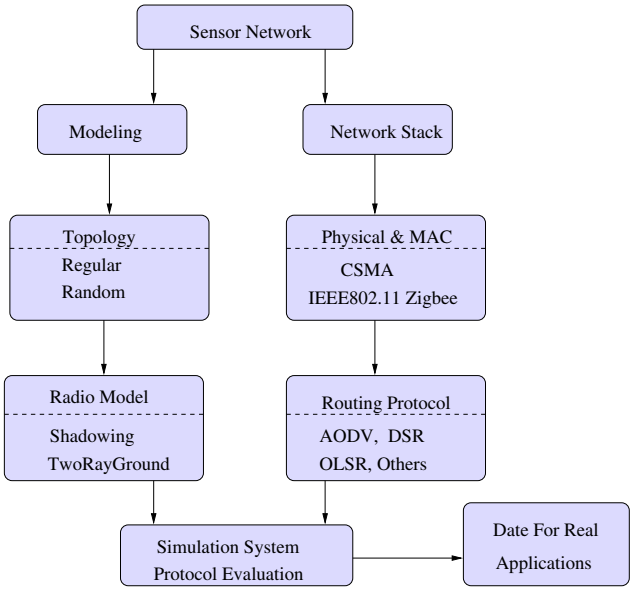

\section{Figure 1. Proposed network simulation model.}

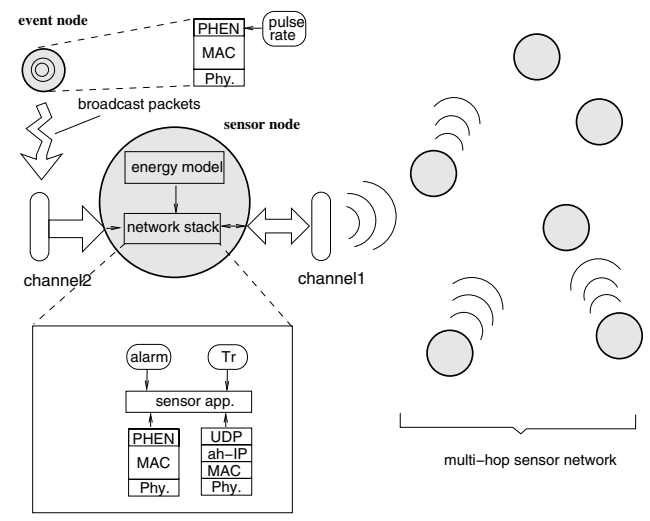

Figure 2. Sensor node model.

For the physical layout of the WSN, two types of deployment has been studied so far: the random and the lattice deployment. In the former, nodes are supposed to be uniformly distributed inside, while in the latter nodes are vertexes of particular geometric shape, e.g. a square grid. In this case, in order to guarantee the connectedness of the network we should set the transmission range of every node to the step size, $d$, which is the minimum distance between two rows (or columns) of the grid. In fact, by this way the number of links that every node can establish, a.k.a the node degree is $D=4$. By using Cooper's theorem [4] along with some power control techniques, one could use also $D=2^{1}$. However, we assume all nodes to be equal and then the degree is fixed to 4 . Nodes at the borders have $D=2$.

\footnotetext{
${ }^{1}$ By using the theorem in [4], we can say that a simple 2 regular network [5] is almost surely strongly 2 connected.
} 


\subsection{Sensor Node and Phenomenon Model}

In order to simulate the detection of a natural event, we used the libraries from Naval Research Laboratory (NRL) [6]. In this framework, a phenomenon is modeled as a wireless mobile node. The phenomenon node broadcasts packets with a tunable synchrony or pulse rate, which represents the period of occurrence of a generic event ${ }^{2}$. These libraries provide the sensor node with an alarm variable. The alarm variable is a timer variable. It turns off the sensor if no event is sensed within an alarm interval. In addition to the sensing capabilities, every sensor can establish a multi-hop communication towards the Monitoring Node (MN) by means of a particular routing protocol. This case is the opposite of the polling scheme. We used two kind of reactive protocols: AODV and DSR.

Sensor node is composed of sensor function model and power model. The sensor function model consists of sensor channel and wireless channel. In the wireless channel is included the physical layer, MAC layer and Network layer. To build the sensor function model, we integrated the sensor protocol stack with the wireless protocol stack.

We assume that the MAC protocol is the IEEE 802.11 standard. This serves us as a baseline of comparison for other contention resolution protocols. The receiver of every sensor node is supposed to receive correctly data bits if the received power exceeds the receiver threshold, $\gamma$. This threshold depends on the hardware ${ }^{3}$. As reference, we select parameters values according to the features of a commercial device (MICA2 OEM). In particular, for this device, we found that for a carrier frequency of $f=916 \mathrm{MHz}$ and a data rate of $34 \mathrm{KBaud}$, we have a threshold (or receiver sensitivity) $\left.\gamma\right|_{d B}=-118 \mathrm{dBm}[7]$. The calculation of the phenomenon range is not yet optimized and the phenomenon propagation is assumed to follows the propagation laws of the radio signals. In particular, the emitted power of the phenomenon is calculated according to a TwoRayGround propagation model [8].

Lattice Network $D=2$ guarantees a connected network. Thus, it suffices to guarantee a value of $\operatorname{Prob}(D \geq 2)$ as close as possible to 1 . Let us note that the link between any two nodes is a bernoullian random variable with a certain probability $p$. If we consider only the closest neighbors, we have that $\operatorname{Prob}(D \geq 2) \geq \sum_{2<k<4}\left(\begin{array}{c}N \\ k\end{array}\right) p^{k}(1-p)^{N-k}$. For $p=0.95, \operatorname{Prob}(D \geq 2) \approx 0.9995$. We can set the maximum transmission range by solving $p=$

\footnotetext{
${ }^{2}$ As a consequence, this model is for discrete events. By setting a suitable value for the pulse rate, it is possible in turn to simulate the continuous signal detection such as temperature or pressure.

${ }^{3}$ Other MAC factors affect the reception process, for example the Carrier Sensing Threshold (CST) and Capture Threshold (CP) of IEEE.802.11 used in NS-2.
}

Table 1. Topology settings.

\begin{tabular}{|c|c|}
\hline \multicolumn{2}{|c|}{ Lattice } \\
\hline \hline Step & $d=\frac{L}{\sqrt{N}-1} \mathrm{~m}$ \\
\hline Service Area Size & $L^{2}=(800 \times 800) \mathrm{m}^{2}$ \\
\hline Number of Nodes & $N=16,64,256$ \\
\hline Transmission Range & $r_{0}=d$ \\
\hline
\end{tabular}

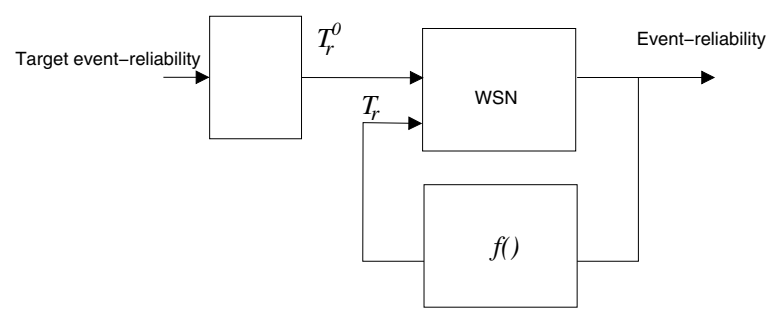
Figure 3. Representation of the transport
based on the event-reliability.

$\operatorname{Prob}\left\{\left.P_{r}(d)\right|_{\mathrm{dB}}>\left.\gamma\right|_{\mathrm{dB}}\right\}=0.95$. It is straightforward to show that:

$$
\begin{aligned}
\left.P_{t}(d)\right|_{\mathrm{dB}} & =\left[10 \alpha \log _{10} d+\gamma||_{\mathrm{dB}}-\operatorname{erfc}^{-1}(2 p) \sqrt{(2)} \sigma\right] \\
& +\beta_{0}
\end{aligned}
$$

where erfc ${ }^{-1}$ is the inverse of the standard error function. Eq. (1) provides the transmission power of each sensor, given a transmission range and a probability or rate of coverage $p$. This should not be confused with the sensing coverage of the ad-hoc WSN.

The distance is not a good link-metric for WSN. In particular, we know that experimental testbeds show a wide range of error probabilities along sensor pairs. The fact that the distance does not correlate well with the error probability is not taken into account by the formula, and it can be considered as a first order approximation.

Interference In general, in every wireless network the electromagnetic interference of neighboring nodes is always present. The interference power decreases the Signalto-Noise-Ratio (SNR) at the intended receiver, which will perceive a lower bit and/or packet error probability. Given a particular node, the interference power depends on how many transmitters are transmitting at the same time of the transmission for the given node. In a ad-hoc WSN, since the number of concurrent transmissions is low because of the low duty-cycle of sensors, we can neglect the interference. In other words, if we define duty-cycle as the fraction between the total time of all transmissions of sensor 
Table 2. Radio model and system parameters.

\begin{tabular}{|c|c|}
\hline \multicolumn{2}{|c|}{ Radio Model Parameters } \\
\hline \hline Path Loss Coefficient & $\alpha=2.7$ \\
\hline Variance & $\sigma_{\mathrm{dB}}^{2}=16 \mathrm{~dB}$ \\
\hline Carrier Frequency & $916 \mathrm{MHz}$ \\
\hline Antenna & omni \\
\hline Threshold (Sensitivity) & $\gamma=-118 \mathrm{~dB}$ \\
\hline \multicolumn{2}{|c|}{ Other Parameters } \\
\hline \hline Reporting Frequency & $T_{r}=[0.1,1000] \mathrm{pps}^{1}$ \\
\hline Interface Queue Size & 50 packets \\
\hline UDP Packet Size & 100 bytes \\
\hline Detection Interval $\tau$ & $30 \mathrm{~s}$ \\
\hline
\end{tabular}

${ }^{1}$ packet per seconds

data and the total operational time of the net, we get always a value less than 0.5. In fact, the load of each sensor is $\ll 1$ because sensors transmit data only when an event is detected [9]. However, it is intuitive that in a more realistic scenario, where many phenomena trigger many events, the traffic load can be higher, and then the interference will worsen the performance with respect to that we study here. Consequently, we can fairly say that the results we get here should be considered as an upper bound on the system performance with respect to more realistic scenarios.

\subsection{Event Detection and Transport}

Here, we use the data-centric model similar to [10], where the end-to-end reliability is transformed into a bounded signal distortion concept. In this model, after sensing an event, every sensor node sends sensed data towards the actor node. The transport used is a UDP-like transport, i.e. there is not any guarantee on the delivery of the data. While this approach reduces the complexity of the transport protocol and well fit the energy and computational constraints of sensor nodes, the event-reliability can be guaranteed to some extent because of the spatial redundancy. The sensor node transmits data packets reporting the details of the detected event at a certain transmission rate ${ }^{4}$. The setting of this parameter, $T_{r}$, depends on several factors, as the quantization step of sensors, the type of phenomenon, and the desired level of distortion perceived at the actor node. In [10], the authors used this $T_{r}$ as a control parameter of the overall system. For example, if we refer to eventreliability as the minimum number of packets required at actor node in order to reliably detect the event, then whenever the actor node receives a number of packets less than the event-reliability, it can instruct sensor nodes to use a higher

\footnotetext{
${ }^{4}$ Note that in the case of discrete event, this scheme is a simple packet repetition scheme.
}

$T_{r}$. This instruction is piggy-backed in dedicated packets from the actor node. This system can be considered as a control system, as shown in Fig. 3, with the target eventreliability as input variable and the actual event-reliability as output parameter. The target event-reliability is transformed into an initial $T_{r}^{0}$. The control loop has the output event-reliability as input, and on the basis of a particular non-linear function $f(\cdot), T_{r}$ is accordingly changed. We do not implement the entire control system, but only a simplified version of it. For instance, we vary $T_{r}$ and observe the behavior of the system in terms of the mean number of received packets. In other words, we open the control loop and analyze the forward chain only.

\section{Routing Protocols}

\subsection{Proactive and Reactive Routing Protocols}

Reactive routing protocols, such as the Dynamic Source Routing (DSR) and Ad-hoc On-demand Distance Vector (AODV) routing protocols, are source-initiated on-demand routing protocols. These types of routing protocols create routes only when requested by the source node. When a node requires a route to a destination, it initiates a route discovery process within the network. This process is completed once a route is found or all possible route permutations have been examined. Once a route is established, it is maintained by a route maintenance procedure either until the destination becomes inaccessible along every path from the source or until the route is no longer needed. This approach can adjust quickly to route changes and does not introduce overhead for periodic control messages when routes are cached or when the network is idle. However, discovering a new route from scratch on demand is costly and bad routes are detected at the cost of packet drops.

Proactive routing protocols, such as the DestinationSequenced Distance-Vector (DSDV), the Topology Broadcast Based on Reverse-Path Forwarding (TBRPF) routing protocols, Optimized Link State Routing (OLSR), maintain up-to-date routing information using periodic control messages. Therefore, proactive routing protocols are ready to exchange packets at anytime. Each node using a proactive routing algorithm maintains one or more tables to store routing information and responds to changes in network topology by propagating updates throughout the network to maintain a consistent view of the network. The areas in which different protocols vary are the number of necessary routing-related tables and the methods by which nodes disseminate changes in network structure. 


\subsection{AODV and DSR Routing Protocols}

We are aware of many proposals of routing protocols for ad-hoc networks. Here, we consider reactive protocols such as AODV and DSR. The AODV and DSR build up a route only when it is needed, i.e. when a node has data to send [11]. In AODV and DSR, there are two phases: Route Request (RREQ) and Route maintenance. The RREQ phase is accomplished by means of broadcast messages to neighbor nodes. In AODV, the destination node chooses one among all possible discovered routes. While in DSR, the source node can learn multiple routes towards the destination. AODV maintains per destination routing tables, while DSR contains multiple routes cache entries for each destination. Moreover, in DSR there is not any mechanism to check whether a cached routed has become staled. For AODV protocol, it is expected that the impact of radio link dynamics is minimal, because of the multi-round mechanism. However, the performance also depends on the infrastructure.

\section{Routing Efficiency}

In this section, we introduce the concept of RE, We consider that after a sensor node detects the physical phenomenon, it sends the packets to the actor node via a routing protocol. The ability to transmit packets for different protocols is different. Also, the RE of a protocol is affected by many network parameters such as wireless transmission radio model, network topology, and transmission frequency. In order to compare the performance of different protocols, we consider the same simulation environment. For our system, we used TwoRayGround radio model and the network topology is regular.

We defined the RE parameter as the ratio of sent packets from sensing node with sent packet by routing protocol. Thus:

$$
R E(\tau)=\frac{N_{\text {sent }}(\tau)}{N_{\text {routing }}(\tau)}
$$

where $N_{\text {routing }}(\tau)$ is the number of sent packets by routing protocol, and $N_{\text {sent }}(\tau)$ is the number of sent packets by sensor nodes which detect the phenomenon. These quantities are computed in a time interval of $\tau$ seconds. For the same simulation time, when RE value is high, the protocol routing efficiency is better. Considering Eq.(2), when $N_{\text {sent }}(\tau)$ value is increased and the $N_{\text {routing }}(\tau)$ value is decreased, the RE is increased. The number of sent packets by sensing node is higher than the number of packet sent by routing protocol. For this reason, the RE function is an increasing function as shown in Fig. 4. The RE is proportional to the transmission rate.

\section{Simulation Results}

In this section, we present the simulation results of our ad-hoc WSN. We simulated the network by means of NS2 simulator, with the support of NRL libraries ${ }^{5}$. For each routing protocol, the sample results of Eq.(2) are computed over 20 simulation runs, and they are plotted in Fig. 4 and Fig. 5. The RE is an increasing function of $T_{r}$, because as $T_{r}$ increases, the number of sent packet by sensing node is higher than the number of packets used by routing protocol. We used two routing protocols: AODV and DSR. In case of AODV, the RE decreases with the increase of number of sensor nodes as shown in Fig. 4. It should be noted that when the number of sensor nodes is increased, then the number of routes is increased, thus the searching time to find a route also is increased. When the number of nodes is 256, the RE of AODV is the worst in our simulation.

In the case of the DSR as shown in Fig. 5, with increase of the number of sensor nodes the RE is also decreased. However, comparing with AODV for the same time interval and for the same number of nodes, the RE of DSR is better. For instance, looking to the simulation results, when the number of nodes is $12, T_{r}=10$, the RE of AODV is 8 , while for DSR the RE is 11 . When there are 256 nodes, the RE of AODV is 0.1 , but the RE of DSR is 0.5 . For $T_{r}=100$, when the number of nodes is 12 , the RE of AODV is 50 , while the RE of DSR is 100 . When the number of nodes is 12 , the RE of DSR is better than AODV. Also, for 256 nodes, the RE of DSR is better than AODV. However, for 12 nodes, the RE of DSR shows fluctuations.

The performance of DSR is better than AODV, because when AODV performs route discovery, it uses the control messages such as RREQ and route reply (RREP). To control the broadcasts of RREQs, the source node uses an expanding ring search technique. When an error happens, the destination node sends a route error (RERR) to the source node and the source sends a new RREQ to the destination node. However, in DSR the source node sends the RREQ with flooding broadcast to the destination node and the destination node reply the RREP. The RREP remembers this request and the address of nodes that have passed to avoid routing again. In DSR, the route cache has more alternative routes and uses more than one mechanism for local route repairs. Thus, DSR invokes the local route repair using the alternative routes in route cache.

\section{Conclusions}

In ad-hoc WSNs, the congestion problem is intermingled with the MAC and routing protocols behavior. In contrast

\footnotetext{
${ }^{5}$ Since the number of scheduler events within a simulated WSN can be very high, we applied a patch against the scheduler module of NS-2 in order to speed up the simulation time [12].
} 


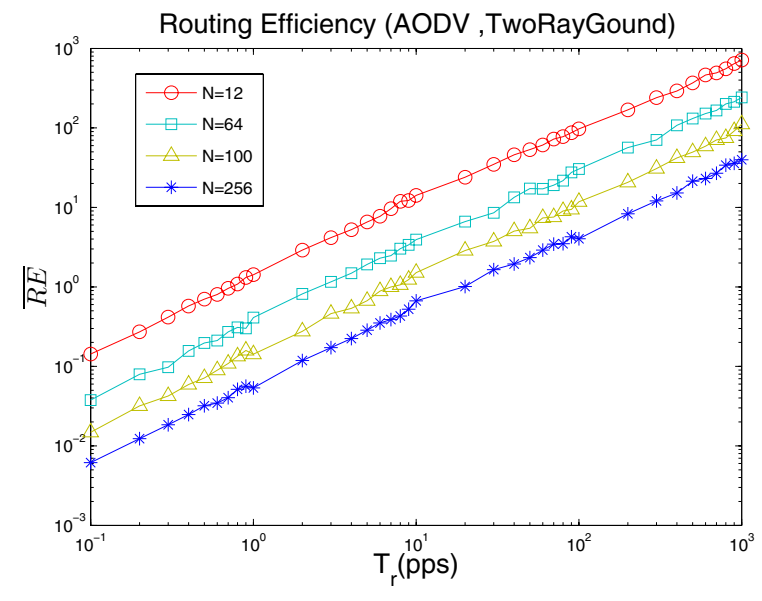

Figure 4. Sample averages of the Routing Efficiency for AODV.

to wired networks, the limited and low capacity of the radio medium, the limited computational and memory capabilities of nodes call for new mechanisms of coordination among sensor nodes. In this work, we analyzed the RE in a square lattice network. We can see the RE of DSR is better than AODV for TwoRayGround radio model.

In the future, we would like to carry out more extensive simulations to evaluate the RE of DSR and AODV. We also plan to evaluate the RE of other protocols such DSDV and OLSR.

\section{Acknowledgment}

This work is partially supported by International Communications Foundation (ICF) of Japan and Japanese Society for the Promotion of Science (JSPS). The authors would like to thank ICF and JSPS for the financial support.

\section{References}

[1] I. F. Akyildiz, I. H. Kasimoglu, "Wireless Sensor and Actor Networks: Research Challenges", Ad Hoc Networks Journal (Elsevier), Vol. 2, No. 4, pp. 351-367, October 2004.

[2] O. Younis, S. Fahmy, "HEED: A Hybrid, Energy-efficient, Distributed Clustering Approach for Ad-hoc sensor Networks", IEEE Transactions on Mobile Computing, Vol. 3, No. 4, pp. 366-379, 2004.

[3] G. W.-Allen, K. Lorincz, O. Marcillo, J. Johnson, and M. Ruiz an J. Lees, "Deploying a Wireless Sensor Network on an Active Volcano", IEEE Internet Computing, Vol. 10, No. 2, pp. 18-25, March 2006.

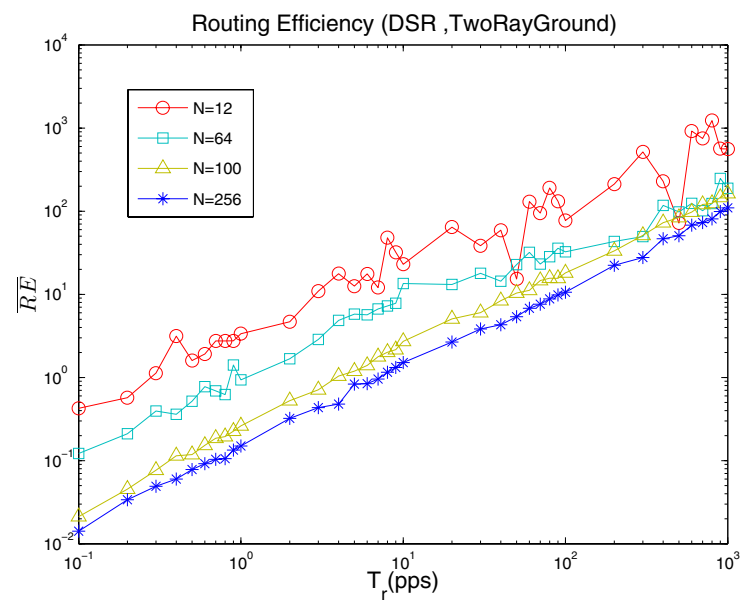

Figure 5. Sample averages of the Routing Efficiency for DSR.

[4] C. Cooper. A Note on the Connectivity of 2-regular Digraphs, Random Structures Algorithms, Vol. 4, pp. 469-472, 1993.

[5] T. Yang, G. De Marco, M. Ikeda, and L. Barolli, "A Case Study of Event Detection in Lattice Wireless Sensor Network with Shadowing-induced Radio Irregularities", Proc. of the 4-th International Conference on Advances in Mobile Computing and Multimedia (MoMM-2006), pp. 241250, 2006.

[6] I. Donward, "NRL's sensor network extension to NS-2", Available on line at http://pf.itd. nrl.navy.mil/nrlsensorsim/, 2004.

[7] Crossbow Technology, Inc., Available on Line at http://www.xbow.com/.

[8] T.S. Rappaport, "Wireless Communications", Prentice Hall PTR, 2001.

[9] W. Ye, J. Heidemann, and D. Estrin, "Medium Access Control with Coordinated Adaptive Sleeping for Wireless Sensor Networks", IEEE/ACM Transaction Networking, Vol. 12, No. 3, pp. 493-506, 2004.

[10] Ö. B. Akan and I. F. Akyildiz, "Event-to-sink Reliable Transport in Wireless Sensor Networks", IEEE/ACM Transactions on Networking, Vol. 13, No. 5, pp. 1003-1016, 2005.

[11] C. Perkins, Editor, "Ad Hoc Networks", Addison-Wesley, 2001.

[12] D. X. Wei. "Speeding up NS-2 Scheduler", Available on Line at http://netlab.caltech.edu/ weixl/technical/ns2patch, California Institute of Technology, September 2005. 